\title{
Author Index Volume 7 (2011)
}

The issue number is given in front of the page numbers.

Aiello, F., G. Fortino, S. Galzarano, R. Gravina and A. Guerrieri, An analysis of java-based mobile agent platforms for wireless sensor networks

(6) $243-267$

Ballet, P., see Rodin, V.

Billhardt, H., R. Centeno, C.E. Cuesta, A. Fernández, R. Hermoso, R. Ortiz, S. Ossowski, J.S. Pérez-Sotelo and M. Vasirani, Organisational structures in next-generation distributed systems: Towards a technology of agreement

Birje, M.N. and S.S. Manvi, An efficient method of sharing device resource status in wireless grids

Bitterberg, T., H. Hildmann and C. Branki, A mobile device based, and multi-agent driven structural design solution

Bosque, J.L., P. Herrero, S. Mata and G. Méndez, Teaching about Madrid: A collaborative agents-based distributed learning course

Branki, C., see Bitterberg, T.

Budimac, Z., see Mitrović, D.

$(2,3) \quad 109-125$

$(4,5) \quad 127-146$

(1) $55-71$

(1) $1-20$

(1) $55-71$

(6) $203-218$

Centeno, R., see Billhardt, H.

Cranefield, S., see Savarimuthu, B.T.R.

$(2,3) \quad 109-125$

(1) $21-54$

Cuesta, C.E., see Billhardt, H.

Czarnul, P., M. Matuszek, M. Wójcik, K. Zalewski, BeesyBees: A mobile agent-based middleware for a reliable and secure execution of service-based workflow applications in BeesyCluster

$(2,3) \quad 109-125$

de Laat, C., see Zhao, Z.

Desmeulles, G., see Rodin, V.

(6) 219-241

(6) $187-202$

$(4,5) \quad 159-182$

Fernández, A., see Billhardt, H.

(2,3) $109-125$

Fortino, G., see Aiello, F.

(6) 243-267

Gal, C.L., see Rodin, V.

$(4,5) \quad 159-182$

Galzarano, S., see Aiello, F.

(6) $243-267$

Gravina, R., see Aiello, F.

(6) $243-267$

Grosso, P., see Zhao, Z.

(6) $187-202$

Guerrieri, A., see Aiello, F.

(6) $243-267$

Hermoso, R., see Billhardt, H.

$(2,3) \quad 109-125$ 
Herrero, P., see Bosque, J.L.

Hexmoor, H., Oversight of reorganization in massive multiagent systems

(1) $\quad 1-20$

Hildmann, H., see Bitterberg, T.

(6) $269-289$

(1) $55-71$

Ivanović, M., see Mitrović, D.

(6) $203-218$

Jemaa, M.B., A fault tolerant platform of web services based on service composition

$(4,5) \quad 147-158$

Koning, R., see Zhao, Z.

(6) $187-202$

Manvi, S.S., see Birje, M.N.

Mata, S., see Bosque, J.L.

Matuszek, M., see Czarnul, P.

McGinnis, J., K. Stathis and F. Toni, A formal model of agent-oriented virtual organisations and their formation

Méndez, G., see Bosque, J.L.

Mendoza, B. and J.M. Vidal, On bidding algorithms for a distributed combinatorial auction

Mitrović, D., Z. Budimac, M. Ivanović and M. Vidaković, Agent-based approaches to managing fault-tolerant networks of distributed multi-agent systems

$(4,5) \quad 127-146$

(1) $1-20$

(6) 219-241

(6) $291-310$

(1) $1-20$

$(2,3) \quad 73-94$

(6) $203-218$

Ontañón, S. and E. Plaza, An argumentation framework for learning, information exchange, and joint-deliberation in multi-agent systems

$(2,3) \quad 95-108$

$(2,3) \quad 109-125$

Ortiz, R., see Billhardt, H.

Ossowski, S., see Billhardt, H.

$(2,3) \quad 109-125$

Pérez-Sotelo, J.S., see Billhardt, H.

$(2,3) \quad 109-125$

Plaza, E., see Ontañón, S.

Rodin, V., G. Desmeulles, P. Ballet, P. Redou and C.L. Gal, An efficient algorithm based on weak synchronization for distributed in virtuo biological experiments

$(4,5) \quad 159-182$

Savarimuthu, B.T.R. and S. Cranefield, Norm creation, spreading and emergence: A survey of simulation models of norms in multi-agent systems

Stathis, K., see McGinnis, J.

(1) $21-54$

(6) $291-310$

Toni, F., see McGinnis, J.

(6) $291-310$

van der Ham, J., see Zhao, Z.

(6) $187-202$

Vasirani, M., see Billhardt, H.

(2,3) $109-125$

Vidaković, M., see Mitrović, D.

(6) $203-218$

Vidal, J.M., see Mendoza, B.

$(2,3) \quad 73-94$

Wójcik, M., see Czarnul, P.

(6) 219-241 
Zalewski, K., see Czarnul, P.

(6) 219-241

Zhao, Z., P. Grosso, J. van der Ham, R. Koning and C. de Laat, An agent based network resource planner for workflow applications

(6) $187-202$ 